\title{
Nonlinear dependence of anomalous resistivity on the reconnecting electric field in the Earth's magnetotail
}

\author{
WU GuiPing* \& ZHANG DaoYu \\ Department of Physics, Southeast University, Nanjing 211189, China \\ Received August 24, 2011; accepted October 25, 2011; published online February 21, 2012
}

\begin{abstract}
Based upon the observational data of the fast magnetic reconnection in the nearly collisionless magnetotail and the particle in cell (PIC) simulations on the electron acceleration in the reconnecting current sheet with guide magnetic field, we self consistently solved one dimension Vlasov equation with the magnetotail parameters and realistic mass ratio to explore the relationship between the anomalous resistivity and the induced electric field. As compared with theoretic formula for the current driven ion-acoustic and Buneman anomalous resistivity, the anomalous resistivity may result from the ion acoustic instability for small reconnecting electric field and the Buneman instability for large reconnecting electric field. The discrepancy between the theoretic results and numerical simulations may be caused by the high frequency instability that results from the deviation of electron distribution from Maxwellian one. These results are consistent with the early experimental results and favorable for the fast reconnection to take place.
\end{abstract}

anomalous resistivity, magnetic reconnection, acceleration of particles

Citation: Wu G P, Zhang D Y. Nonlinear dependence of anomalous resistivity on the reconnecting electric field in the Earth's magnetotail. Chin Sci Bull, 2012, 57: 1449-1454, doi: 10.1007/s11434-011-4902-4

In a collissioness coronal and the Earth's magnetotail plasma, anomalous resistivity caused by kinetic-scale wave-particle interactions is usually assumed to trigger fast magnetic reconnection, which was widely accepted as the main mechanism that converts free magnetic energy into heating and accelerating electrons and produces magnetospheric substorms [1-4]. The energetic electrons up to $\sim 300 \mathrm{keV}$ were measured in a rare crossing of the diffusion region of reconnecting current sheet (RCS) by the Wind spacecraft [5]. Egedal et al. [6], after analyzing in-situ measurements of electron distribution functions inside the magnetotail RCS respectively from Wind and Cluster, found that the relative energy gain of the superthermal electrons is the same and nearly independent of their initial energy, while the electrons with the thermal energy are less accelerated. Hence, they concluded that the super thermal electrons are accelerated by reconnecting parallel electric field in vicinity of the reconnection region. For other acceleration mechanisms, such as Fermi acceleration of electrons in contracting magnetic islands and stochastic acceleration, the

*Corresponding author (email: wuguiping@ @eu.edu.cn) relative energy gain of the electrons is a function of their initial energy. Therefore, these direct observational signatures suggest that the electrons are accelerated to relativistic energy by reconnecting electric field inside the diffusion region.

On the other hand, the role of the electron dynamics inside the diffusive region of RCS is very important to understand the collissionless magnetic reconnection [7]. The 2D and 3D particle-in-cell (PIC) simulations was widely used to trace the typical electron trajectories $[8,9]$. It is found that, with the guiding magnetic field or external driven reconnection in RCS, the electrons can only be accelerated by the electric field in the X-type region and then spread out along the separatrices. In the O-type region, no obvious electron acceleration is observed.

For the generation of anomalous resistivity $\eta_{\mathrm{eff}}$, it was often assumed that the current driven electrostatic ion-acoustic instability was excited after a shrinkage of the current sheet in the resistive MHD simulations. If the current density is larger than the threshold of ion-acoustic instability, the resistivity suddenly increases several orders above the classic Spitz resistivity. Using its relation to the effective collision 
frequency $v_{\mathrm{eff}}$ in the presence of current driven ion-acoustic waves, i.e. $\eta_{\mathrm{eff}}=v_{\mathrm{eff}} /\left(\varepsilon_{0} \omega_{p e}^{2}\right)[10]$, we have

$$
\eta_{\mathrm{eff}}=\frac{1}{\varepsilon_{0} \omega_{p e}} \frac{\varepsilon_{0} \delta E^{2}}{2 n_{e} \kappa_{B} T_{e}},
$$

where $\omega_{p e}$ is the electron plasma frequency, $n_{e}$ is the electron number density, $T_{e}$ is the electron temperature, and $\varepsilon_{0} \delta E^{2} / 2$ is the observed wave power of the fluctuations.

Recently, one dimension (1D) Vlasov simulations with parameters appropriate to the magnetopause and low-latitude boundary layer highlight the importance of the anomalous resistivity produced by the ion acoustic instability [11]. Petkaki and Freeman inferred non-linear dependance of anomalous resistivity on the bulk drift velocity of electrons [12]. We performed 1D Vlasov simulations, including the external inductive field with a realistic mass ratio and parameters appropriate to solar coronal for the first time, investigated the nonlinear dependance of the anomalous resistivity on the induced electric field and bulk drift velocity, and found that the excited electrostatic waves have the characteristics of the Buneman instability, i.e. propagating in opposite directions with the growth rate of the order of the ion plasma frequency, and decreasing the wave vector at the maximum growth rate with increase of the bulk drift velocity of electrons [10-12].

As the anomalous resistivity is sensitive to the plasma parameters and the initial condition, we investigate the nonlinear dependance of anomalous resistivity on the induced electric field by means of Vlasov simulations with the parameters direct measurement from the fast reconnection in the magnetotail by Cluster [3]. The time dependent evolution of electron distribution and the energy of unstable waves are also presented.

\section{Basic equation and simulation method}

There are two approaches in the present work: (1) the reconnecting and perpendicular components of the magnetic field approach zero, i.e. $B_{x} \approx B_{y} \approx 0$, which are appropriate in the center-plane of a current sheet near the X-type point with guiding field, where the electrons are most effectively accelerated [5,6]; (2) the Lorentz force $(\boldsymbol{J} \times \boldsymbol{B})$ is smaller than the electric force, when the induced electric field is assumed to be along the $z$-component of the magnetic field $[8,10]$. Therefore, for the study of electrostatic waves, the only force acting on the plasma is that of an electric field, a 1D approach may be enough to investigate the evolution of the anomalous resistivity due to the current-driven electrostatic instability in RCS $[8,10,13]$.

The 1D electrostatic Vlasov equation is written as

$$
\frac{\partial f_{\alpha}}{\partial t}+v_{z} \frac{\partial f_{\alpha}}{\partial z}+\frac{q_{\alpha}}{m_{\alpha}} E_{z} \frac{\partial f_{\alpha}}{\partial v_{z}}=0
$$

where $f_{\alpha}$ is the particle distribution function $(\alpha \in\{i, e\}), m_{\alpha}$ and $q_{\alpha}$ are respectively the mass and charge of particles, $E_{z}$ is the electric field strength, including the inductive component $E_{0}$ and turbulent component $\widehat{E}_{z}$, i.e. $E_{z}=E_{0}+\widehat{E}_{z}$. The former is assumed to be a constant in time and space, and the latter may be integrated forward in time, using Ampere's law given by [17]

$$
(\nabla \times \boldsymbol{B})_{z}=\mu_{0}\left(J+\varepsilon_{0} \frac{\partial \widehat{E}_{z}}{\partial t}\right) .
$$

The electric current density is expressed by

$$
J(z, t)=\sum q_{\alpha} \int v_{z} f_{\alpha}\left(z, v_{z}, t\right) \mathrm{d} v_{z},
$$

which may be divided into two parts of a spatially-averaged component and a fluctuant component, the former being assumed to be balanced by the gradient of an external magnetic field $\boldsymbol{B}$ at all time, i.e. $(\nabla \times \boldsymbol{B})_{z}=\mu_{0}\langle J\rangle[11]$, and the latter being related to the turbulent electric field, which is given by $\widehat{J}=J-\langle J\rangle$.

The anomalous resistivity may be calculated at each time step using the following expression [14]:

$$
\eta_{\mathrm{eff}}=\frac{E_{0}}{\langle J\rangle}-\frac{m_{e}}{n_{e} e^{2}}\left(\frac{1}{\langle J\rangle} \frac{\mathrm{d}\langle J\rangle}{\mathrm{d} t}\right) .
$$

With the periodic boundary conditions and simulation method described in Horne and Freeman [17], eq. (2) is integrated forward in time, with initial unstable waves originated in a white noise electric field applied at $t=0$ [18]. In our simulations, the ion to electron mass ratio is $m_{p} / m_{e}=1836$, $n_{i}=n_{e}=2 \times 10^{5} \mathrm{~m}^{-3}$ [3], $T_{e}=T_{i}=100 \mathrm{eV}$ [19], where $n_{i}$ is the ion number density, $T_{i}$ is the ion temperature. According to a multi-spacecraft measurement of fast reconnection rate $\gamma_{\text {rec }} \sim V_{\text {in }} \approx(0.07 \sim 0.15) V_{A}$ and $B_{0}=18.9$ nT [3], the induced electric field strength may be estimated as $E_{0}=V_{\text {in }} \times B_{0}=(0.862 \sim 4.13) \times 10^{-3} \mathrm{~V} \mathrm{~m}^{-1}$. We take $E_{0}=(0.1 \sim 5) \times 10^{-3} \mathrm{~V} \mathrm{~m}^{-1}$. The number of grid points is $N_{z} \times N_{v e} \times N_{v i}=2000 \times 2500 \times 400$. The resolution of spacial grid $\Delta z$, velocity grid $\Delta v_{e}$ and $\Delta v_{i}$ are respectively $0.5 \lambda_{D e}, 0.01 v_{e 0}, 0.05 v_{i}$, where $v_{i}$ and $v_{e 0}$ are respectively the ion and initial electron thermal velocity $\left(v_{i}=\sqrt{k T_{i} / m_{i}}\right.$, $v_{e o}=\sqrt{k T_{e} / m_{e}}$ ), and $\lambda_{D e}$ is the plasma Debye length. The time step is $\Delta t=0.003 w_{p e}^{-1}$. The space, velocity space, and time numbers of grid points are carefully selected to ensure the numeric stability and accuracy of the integration algorithm $[17,18]$. The initial ion and electron populations are respectively drift-like Maxwellian distribution functions, $f_{i}=$ $n_{i} /\left((2 \pi)^{1 / 2} v_{i}\right) \exp \left(-v^{2} /\left(2 v_{i}^{2}\right)\right), f_{e}=n_{e} /\left((2 \pi)^{1 / 2} v_{e 0}\right) \exp (-(v-$ $\left.v_{d}\right)^{2} /\left(2 v_{e 0}^{2}\right)$ ), where $v_{d}$ is the initial bulk drift velocity of electrons relative to ions. As the critical drift velocity for the onset of ion-acoustic instability with $T_{e} / T_{i}=1$ is about $1.35 v_{e 0}$, we take $v_{d} / v_{e 0}=1.5$ to save computing time [15].

\section{The nonlinear dependance of the anomalous resistivity on the induced electric field}

\subsection{Evolution of the electron distribution}

The electron distribution at $z=0$, the spatially averaged mean drift velocity, thermal velocity, free accelerated veloc- 
ity of electrons with different magnitudes of external inductive fields are respectively shown in Figures 1 and 2. It can be seen that the whole evolution can be divided into three stages. At the beginning, the electrons are all accelerated by the induced electric field, and Buneman instability is excited. Then, with the increase in the magnitude of unstable waves, the kinetic energy of electrons is transferred into waves, and the drift velocity decreases. As the unstable waves propagate opposite directions and some electrons are scatted into a positive region of $v_{z}$-axis, a low-velocity tail appears relative to the initial distributions and trapped by waves in Figure 1, and their acceleration stops. Finally, after the instability saturates, the untrapped electrons continue to be accelerated, and the bulk drift velocity continues to rise $[13,20]$.

\subsection{Anomalous resistivity}

As stated in [12] that the anomalous resistivity during the nonlinear phase is highly variable in time, and sensitive to the initial noise fields, we numerically solved eqs. (2)-(5) for nine different induced electric fields, and used an ensemble of 10 Vlasov simulations for each set of initial conditions, which differ only in the initial electric field noise as that in [12]. The time evolution of the ensemble Vlasov simulations for $E_{0}=0.00025 \mathrm{~V} \mathrm{~m}^{-1}, 0.0025 \mathrm{~V} \mathrm{~m}^{-1}$ are plotted in Figure 3, where thin lines mark each $\eta$ of the 10 initial noise fields and thick line denote mean $\eta$. Figure 4 shows the mean of peak value $\bar{\eta}_{\max }$ (plus marks), and the standard deviation $\sigma\left(\bar{\eta}_{\max }\right)$
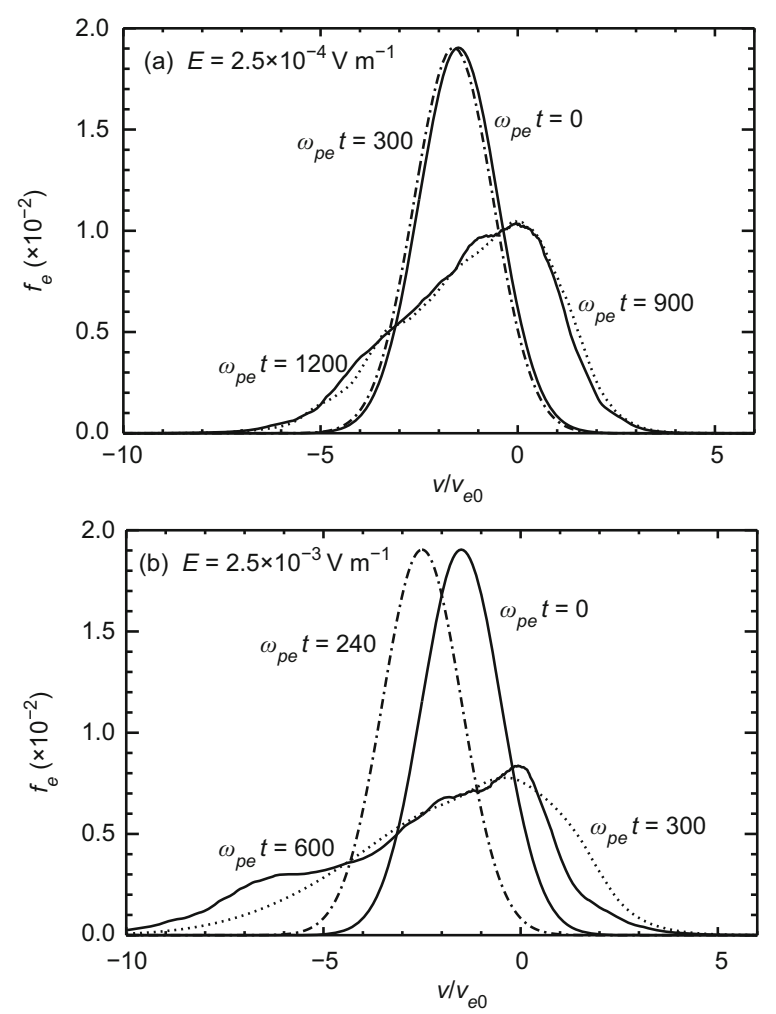

Figure 1 Evolution of the electron distribution for two induced electric fields.
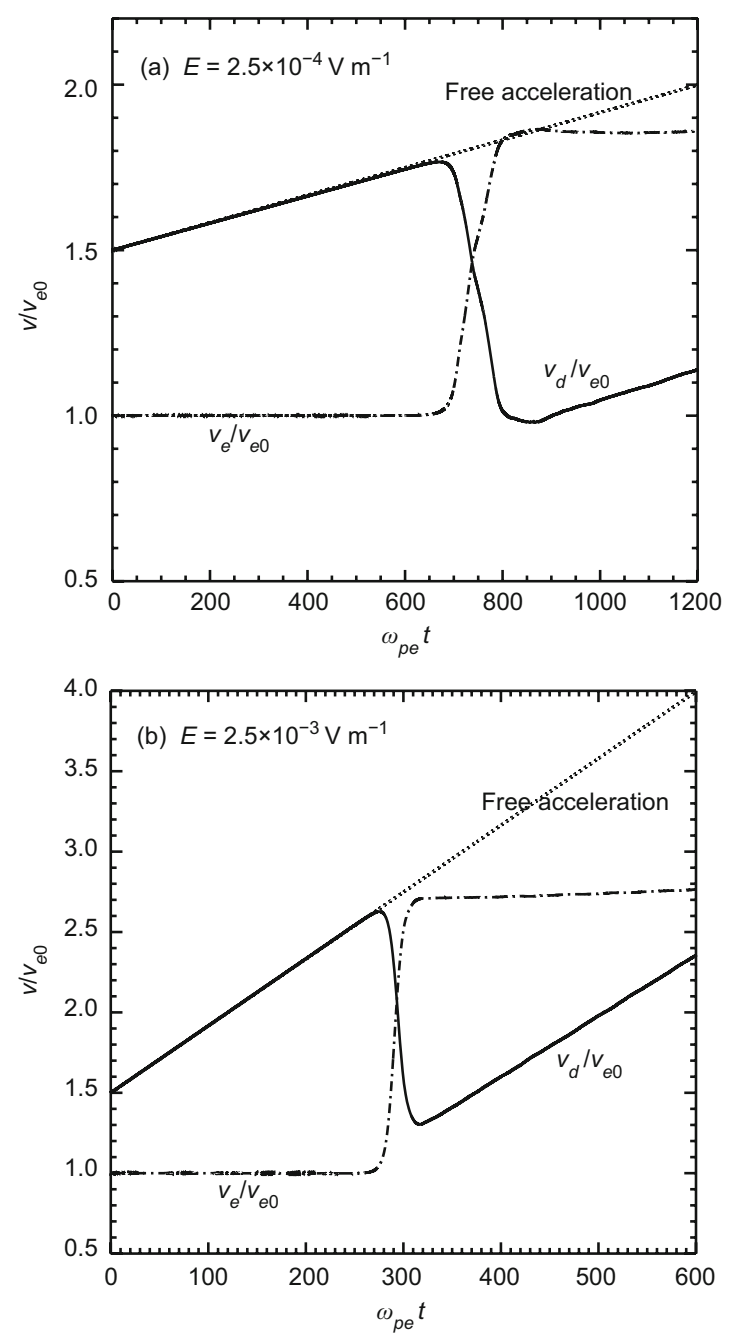

Figure 2 Evolution in bulk drift velocity, thermal velocity, and free accelerated velocity of electrons for two different induced electric fields.

(dotted lines).

Previously, eq. (1) is usually used to estimate the anomalous resistivity due to wave-particle scattering. Substituting the typical values into eq. (1), we have $\eta_{\mathrm{eff}}=4.47 \times 10^{8} \sigma_{e}$, where $\sigma_{E}=\left(\varepsilon_{0} \hat{E}^{2}\right) /\left(2 n_{e} k T\right)$ is the ratio of the turbulent wave energy to the thermal energy. Substituting the spatially averaged value of $\sigma_{E}$ versus time into eq. (1), we plot the evolution of the anomalous resistivity, and compare it with the one from eq. (5) in Figure 5. It can be seen that: (1) in the rising phase of unstable waves, the anomalous resistivity obtained in two ways has the same order; (2) in the maximum phase of unstable waves, the difference between them increases with enhancement of $E_{0}$; (3) after saturation of unstable waves, because the energy exchanges among the waves and particles stochastically, the averaged total energy of unstable waves or particles almost changes little, and the electron distribution in velocity space also hardly changes. Since the wave-particle interaction is very dynamic, eq. (1) is too simple to describe the evolution of anomalous resistivity.

As further compared with the classic resistivity due to Coulomb collisions, the peak value of the anomalous resistiv- 

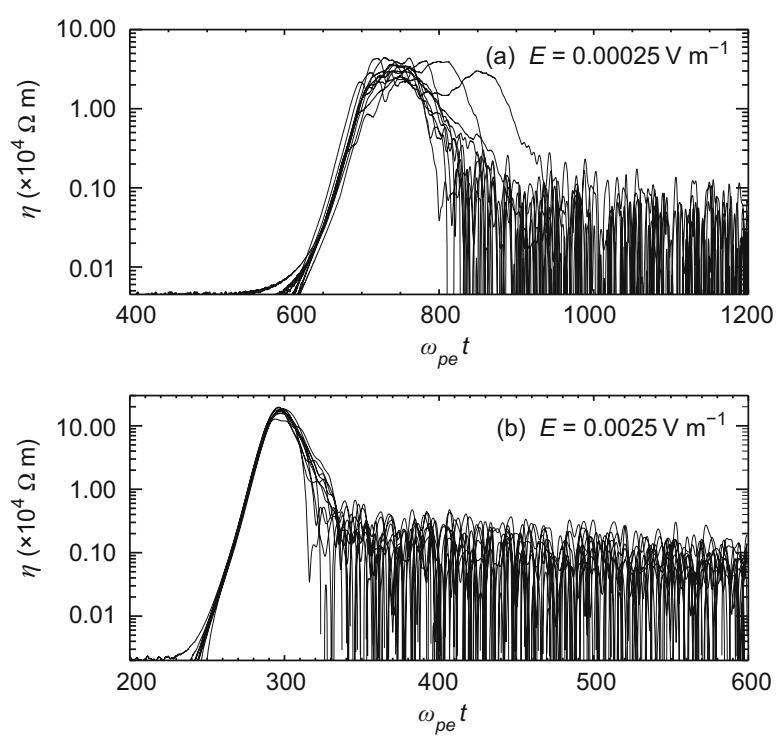

Figure 3 Anomalous resistivity plotted as a function of time, where thin lines mark each of the 10 initial noise fields and thick line denote mean $\eta$.

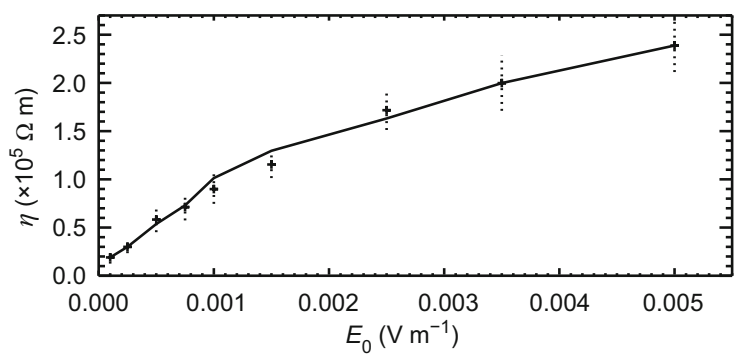

Figure 4 Anomalous resistivity plotted as a function of the induced electric field.

ity enhances about ten orders for $E_{0}=10^{-4} \mathrm{~V} \mathrm{~m}^{-1}$, which can be calculated from formula of $\eta \approx 10^{-3} T_{e}^{-\frac{3}{2}}(\mathrm{eV})=10^{-6} \Omega \mathrm{m}$ with $T_{e}=100 \mathrm{eV}$. After saturation of the unstable waves, the peak value decreases at least two orders. Martens and Yong also estimated the anomalous resistivity in Geomagnetic tail, which enhances about nine orders as compared with the classic resistivity [19].

In addition, the early experiments also told us that if the applied electric field is relatively small, the anomalous conductivity is associated with ion-acoustic instability, if the applied electric field is large, the anomalous conductivity is associated with Buneman instability. Substituting the typical coronal parameters into the formulas $\sigma(e s u) \approx\left(\frac{m_{p}}{m_{e}}\right)^{\frac{1}{3}} \frac{\omega_{p e}}{2}$ for the anomalous current driven Buneman conductivity and $\sigma(e s u) \approx\left(\frac{m_{p}}{m_{e}}\right)^{\frac{1}{2}} \omega_{p e}$ for the anomalous current driven ionacoustic conductivity [21], we have $7.8 \times 10^{4} \Omega \mathrm{m}$ for anomalous Buneman resistivity, and $0.83 \times 10^{4} \Omega \mathrm{m}$ for anomalous ion acoustic resistivity. From our simulations, the anomalous resistivity calculated is about $(1.9-3) \times 10^{4} \Omega \mathrm{m}$ for $E_{0}=(1-$ $2.5) \times 10^{-4} \mathrm{~V} \mathrm{~m}^{-1}$ (Figure 4), and (8.99-17.2) $\times 10^{4} \Omega \mathrm{m}$ for $E_{0}=(1-2.5) \times 10^{-3} \mathrm{~V} \mathrm{~m}^{-1}$ (Figure 4$)$, which is the same order as the theoretical one respectively from ion and Buneman instability. As we have demonstrated that the interaction of
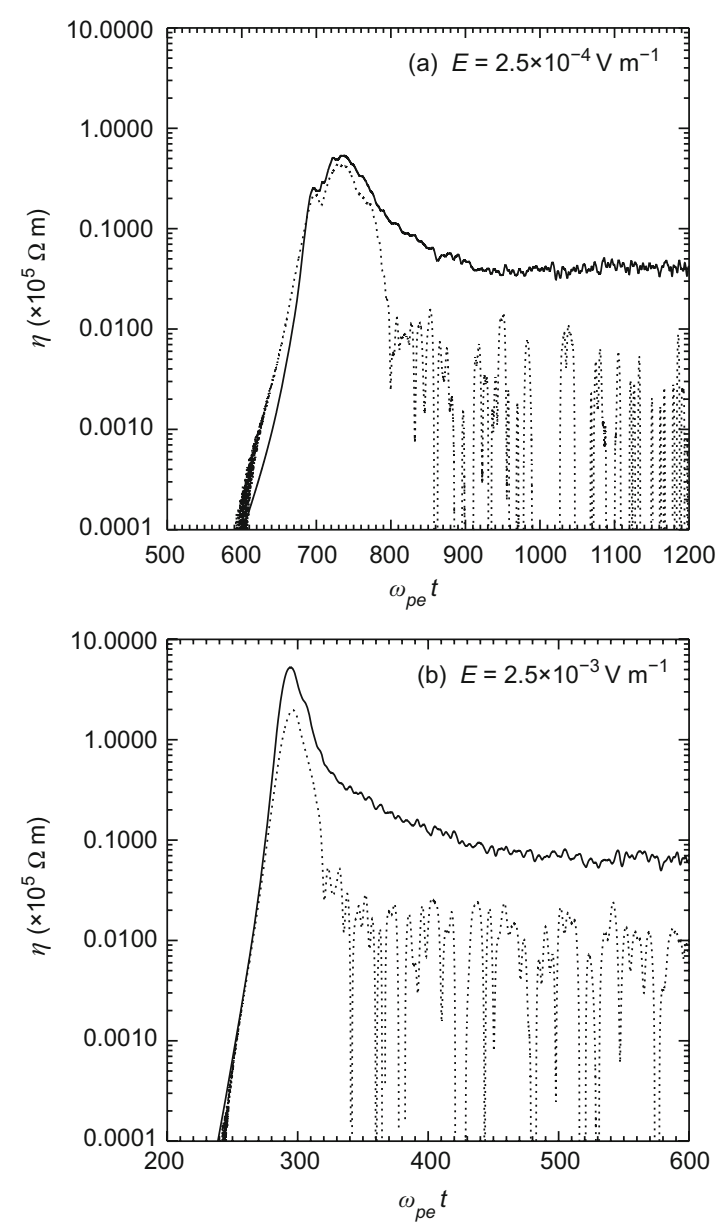

Figure 5 Comparing the anomalous resistivity calculated respectively from eqs. (1) and (5), where solid line marks the one from eq. (1) and dotted line denotes the one from eq. (5).

wave and electrons lead the distribution of electrons deviates from Maxwellian one (see Figure 1), the beam-plasma instabilities are excited [13], which may be the reason for the enhancement of anomalous resistivity with the increase of the inductive electric field. Therefore, our numerical simulations are basically consistent with these experimental results for different electric fields.

\section{Conclusions and discussion}

Based upon the observations and PIC simulations that in the Earth's magnetotail, the energetic electrons are accelerated by the induced reconnecting electric field near the center of RCS with guiding magnetic field, we performed 1D Vlasov simulation including the reconnecting electric field with parameters of Earth's magnetotail and real mass ratio of electron to ion, investigated the nonlinear evolution of electron distribution, and self consistently worked out the anomalous resistivity due to wave-particle scatting. The main results are summarized as follows.

(1) At first, owing to the presence of a super-Dreicer electric field [19], the electrons are freely accelerated and the 
bulk drift velocity increases. Then, with the excitation of current driven instability, wave-particle scatting causes the low-velocity tail to be formed and the bulk drift velocity decreases. Finally, those electrons trapped by waves stop accelerating, and the other untrapped electrons continue to be accelerated. The bulk drift velocity increases.

(2) Substituting the parameters into theoretic formula respectively for current driven ion-acoustic resistivity and Buneman resistivity, we have similar simulation results respectively for small and large $E_{0}$. It demonstrates that anomalous resistivity may result from the current driven ion acoustic instability for small $E_{0}$ and Buneman instability for large $E_{0}$, respectively. The discrepancy may caused by the high frequency instability that results from the deviation of electron distribution from Maxwellian one [13]. These results are also consistent with early experimental results.

(3) In the rise phase of unstable waves, the anomalous resistivity is the same order as the one estimated from quasi linear theory. In the maximum phase of unstable waves, the difference between them increases with enhancement of $E_{0}$. After saturation of unstable waves, the anomalous resistivity decrease at least about 2-3 orders as compared with its peak value. Since the wave-particle interaction is very dynamic, eq. (1) is too simple to describe the evolution of anomalous resistivity.

A number of numerical resistive MHD simulations demonstrated that not only the magnitude of anomalous resistivity but also its distribution decide reconnection rate. The main conclusions are that: (1) a spatially uniform resistivity triggers the Sweet-Parker reconnection to take place; (2) a locally enhanced resistivity inside the diffusive region near the center of X-type neutral point leads Petschek-like reconnection to take place, and the fast reconnection drastically evolves by a positive feedback between the global reconnection flow and the anomalous resistivity [22,23].

In principle, the anomalous resistivity and its distribution in RCS may be fully understood only when the 3D selfconsistent dynamic reconnection is solved. However, due to the limit of the run time and storage memory of computers, a simplified model and unrealistic plasma parameters are often used to get some insight of its physical nature. Since the most efficient electron acceleration in RCS takes place near the X-line with externally driven RCS with guiding magnetic field $[8,9]$, it suggests that the anomalous resistivity larger near the neutral point than the one in the other region, which is favorable for the fast magnetic reconnection to take place.

Considering that the energetic particles in tenuous and collisionless space plasmas are better fitted by kappa-type distributions [18, 24-26], we substituted the initial kappa-type ones into our simulations with $E_{0}=0.0025 \mathrm{~V} \mathrm{~m}^{-1}$, obtained that the averaged peak anomalous resistivity is respectively $(1.50,1.62,1.72) \times 10^{5} \Omega \mathrm{m}$, and the the corresponding peak time is almost the same for the index $\kappa=3,4, \infty$ (Maxwellian distribution, see eq. (1) in [18]). It represents that the impact is small, which may be caused by the deviation of the elec- tron distribution from the Maxwellian one after the onset of the unstable waves. The difference for these two distributions in exciting the instability is the lower threshold of drift velocity for kappa-type one [18]. On the other hand, the more unstable waves are excited in a multi dimensional simulations, which may play an important role in heating and accelerating electrons in some stage [20,27].

In addition, we emphasize that the distribution of the energetic electrons in RCS could not be obtained by the present 1D simulation, because the small magnetic field component perpendicular to the guiding field is ignored, which causes the electrons to move outside the diffusion region without further acceleration. Only when 3D electromagnetic simulation in RCS is performed, can the self-consistent energetic electron spectrum be acquired.

We are grateful to the referees for their constructive comments and suggestions on our work and manuscript. We would like to acknowledge the computational resources provided by the Department of Physics, Southeast University. This work was supported by the National Natural Science Foundation of China (11073006).

1 Rong Z J, Shen C, Lucek E, et al. Statistical survey on the magnetic field in magnetotail current sheets: Cluster observations. Chin Sci Bull, 2010, 55: 2542-2547

2 Øieroset M, Phan T D, Fujimoto M, et al. In situ detection of collisionless reconnection in the Earth's magnetotail. Nature, 2001, 412: 414-417

3 Xiao C J, Pu Z Y, Wang X G, et al. A cluster measurement of fast magnetic reconnection in the magnetotail. Geophys Res Lett, 2007, 34: L01101

4 Chu X N, Pu Z Y, Cao X, et al. THEMIS observations of two substorms on February 26, 2008. Sci China Tech Sci, 2010, 53: 1328-1337

5 Øieroset M, Lin R P, Phan T D, et al. Evidence for electron acceleration up to $\sim 300 \mathrm{keV}$ in the magnetic reconnection diffusion region of the Earths magnetotail. Phys Rev Lett, 2002, 89: 195001

6 Egedal J, Lê A, Zhu Y, et al. Cause of superthermal electron heating during magnetotail reconnection. Geophys Res Lett, 2010, 37: L10102

7 Lu Q M, Wang R S, Xie J L, et al. Electron dynamics in collisionless magnetic reconnection. Chin Sci Bull, 2011, 56: 1174-1181

8 Fu X R, Lu Q M, Wang S. The process of electron acceleration during collisionless magnetic reconnection. Phys Plasmas, 2006, 13: 012309

9 Pritchett P L. Relativistic electron production during driven magnetic reconnection. Geophys Res Lett, 2006, 33: L13104

10 Labelle J, Treumann R A. Plasma waves at the dayside magnetopause. Space Sci Rev, 1988, 47: 175-202

11 Watt C E J, Horne R B, Freeman M P. Ion-acoustic resistivity in plasmas with similar ion and electron temperatures. Geophys Res Lett, 2002, 29: L1004

12 Petkaki P, Freeman M P. Nonlinear dependence of anomalous ionacoustic resistivity on electron drift velocity. Astrophys J, 2008, 686: 686-693

13 Wu G P, Huang G L. Electron acceleration in the turbulent reconnecting current sheets in solar fares. Astron Astrophys, 2009, 502: 341-344

14 Wu G P, Huang G L, Ji H S. Dependence of anomalous resistivity on the induced electric field in solar fares. Astrophys J, 2010, 720: 771-774

15 Wu G P, Huang G L, Ji H S. Dependence of anomalous resistivity on bulk drift velocity of electrons in the reconnecting current sheets in solar fares. Res Astron Astrophys, 2010, 11: 1186-1194 
16 Omura Y, Heikkila W J, Umeda T, et al. Particle simulation of plasma response to an applied electric field parallel to magnetic field lines. J Geophys Res, 2003, 108A: 1197

17 Horne R B, Freeman M P. A new code for electrostatic simulation by numerical integration of the Vlasov and Ampere equations using MacCormack's method. J Comp Phys, 2001, 171: 182-200

18 Petkaki P, Watt C E J, Horne R B, et al. Anomalous resistivity in nonMaxwellian plasmas. J Geophys Res, 2003, 108: 1442

19 Martens P C H, Young A. Neutral beams in two-ribbon flares and in the geomagnetic tail. Astrophys J Suppl Ser, 1990, 73: 333-342

20 Che H, Drake J F, Swisdak M, et al. Nonlinear development of streaming instabilities in strongly magnetized plasmas. Phys Rev Lett, 2009, 102: 145004-145007

21 Hamberger S M, Friedman M. Electrical conductivity of a high turbulent plasma. Phys Rev Lett, 1968, 21: 674-677
22 Yokoyama T, Shibata K. What is the condition for fast magnetic reconnection. Astrophys J, 1994, 436: L197-200

23 Ugai M, Zheng L. Conditions for the fast reconnection mechanism in three dimensions. Phys Plasmas, 2005, 12: 092312

24 Xiao F L. Modelling energetic particles by a relativistic kappa-loss-cone distribution function in plasmas. Plasma Phys Control Fusion, 2006, 48: 203-213

25 Xiao F L, Chen L X, Li J F. Energetic particles modeled by a generalized relativistic kappa-type distribution function in plasmas. Plasma Phys Control Fusion, 2008, 50: 105002

26 Xiao F L, Shen C L, Wang Y M, et al. Energetic electron distributions fitted with a relativistic kappa-type function at geosynchronous orbit. J Geophys Res, 2008, 113: A05203

27 Xiao F L, Zong Q G, Pu Z Y, et al. Electron acceleration by whistlermode waves around the magnetic null during 3D reconnection. Plasma Phys Control Fusion, 2010, 52: 052001

Open Access This article is distributed under the terms of the Creative Commons Attribution License which permits any use, distribution, and reproduction in any medium, provided the original author(s) and source are credited. 